\title{
A new tool for predicting in-hospital mortality in Chinese older patients with exacerbation of chronic obstructive pulmonary disease(AECOPD): frailty index based on common laboratory tests (FI-Lab)
}

Jinjin Gu

Zhenjiang First People's Hospital

Lijie Zheng

Zhenjiang First People's Hospital

Qiang Liu ( $\square$ jenny_2007_hi@163.com )

affiliated hospital of Jiangsu university https://orcid.org/0000-0003-2108-121X

\section{Research}

Keywords: frailty index, FI-Lab, DECAF, AECOPD, prognosis

Posted Date: December 7th, 2020

DOI: https://doi.org/10.21203/rs.3.rs-121756/v1

License: (c) (i) This work is licensed under a Creative Commons Attribution 4.0 International License.

Read Full License 


\section{Abstract}

Introduction: The exacerbation of chronic obstructive pulmonary disease(AECOPD)is a common and fetal disease but with no ideal predictor of in-hospital mortality. Frailty prevails in older adults with AECOPD and can cause increased vulnerability to many adverse health outcomes including death. However, we know little about how frailty affects in-hospital mortality in older AECOPD patients.

Objective: To explore the predictive validity of Fl-Lab-an objective tool for assessing frailty including 21 routine blood tests plus systolic and diastolic blood pressure (a score between 0 and 1, a higher score indicates greater frailty) - for in-hospital mortality in patients with AECOPD.

Methods: We reviewed the hospitalization records of older AECOPD inpatients from September 2016 to June 2019 at Zhenjiang First People's Hospital. We compared survivors to non-survivors. We used propensity score matching (PSM) to balance priori differences between survivors and non-survivors. Logistic regression analysis was used to select the associated predictors of in-hospital mortality. Receiver-operating characteristic (ROC) curves were calculated to estimate the area under the ROC curve (AUCs) for FI-Lab and DECAF(a commonly used predictor of AECOPD including dyspnea, eosinophilia, pulmonary consolidation, acidemia, and atrial fibrillation; a score between 1 and 6; a

higher score indicates poorer condition)in relation to mortality. Data were analyzed using IBM SPSS for Windows, Version 23.0.

Results: A total of 154 patients -77 survivors and 77 non-survivors - were included in the study finally. The mean age of these patients was $79.73 \pm 8.38$ years. Both of the mean DECAF score, the mean FI-Lab value of non-survivors were statistically higher than those of survivors $(4.45 \pm 0.80$ versus $3.03 \pm 0.90, P=$ $0.000 ; 0.51 \pm 0.13$ versus $0.29 \pm 0.10, P=0.000$,respectively). Logistic regression analysis suggested that high DECAF grade and high FI-Lab grade were strong related factors of death in AECOPD patients (OR:5.620,95\% Cl 2.811-11.236, $P=0.000 ; \mathrm{OR}: 8.705,95 \% \mathrm{Cl} 3.646-20.782, P=0.000$, respectively). The DECAF scores of most non-survivors were $\geq 4 \llbracket n=71,92.21 \% \rrbracket$. FI-Lab value predicted in-hospital mortality at a cut-off value of 0.4388 with $70.1 \%$ sensitivity, $96.1 \%$ specificity, 0.675 Youden index. DECAF score predicted in-hospital mortality at a cut-off value of 3.5 with $92.2 \%$ sensitivity, $72.7 \%$ specificity, 0.649 Youden index. The areas under the ROC curves were 0.906 for FI-Lab and 0.870 for DECAF with no statistically significant $P=0.2991$ ).

Conclusions: FI-Lab has a slightly stronger screening ability than DECAF. FI-Lab is a simple, effective and objective indicator and can be quick to help clinicians to assess in-hospital mortality of AECOPD patients.

\section{Introduction}

Chronic obstructive pulmonary disease (COPD) is a chronic, common and fetal disease. Acute exacerbation of chronic obstructive pulmonary disease (AECOPD) in the older adults indicates declining physical activity and deteriorating lung function[1]. Assessing in-hospital mortality risk rapidly and 
accurately by a simple prognostic marker will be helpful in grading nursing care and allocating medical resources. Current prognostic markers about AECOPD include two main categories: inflammation-related makers[2-4] and DECAF score[5-7]. Inflammatory mediators can destroy lung structure and promote neutrophil-related inflammation, which can aggravate the disease and cause death. Inflammation-related markers such as C-reactive protein (CRP) and interleukin 6 [2, 4], red blood cell distribution(RDW) width[8, 9], eosinophil count[10], and neutrophil and lymphocyte ratio (NLR) [11] are often associated with mortality or readmission in AECOPD patients. The DECAF score $[5,7,12]$ (including dyspnea, eosinophilia, pulmonary consolidation, acidemia, and atrial fibrillation) is a commonly used predictor of in-hospital mortality in AECOPD patients.

However, most of the Inflammation-related markers can only suggest significant changes between AECOPD patients and stable COPD patients but display relatively poor value in prognosis. DECAF scoring system needs five complex indicators: clinical presentation, electrocardiogram, imaging(chest X-ray or CT scan), blood gas analysis, and routine blood tests. And it is subjective to assess clinical presentation including degree of dyspnea and difficult to judge whether or not there are pulmonary consolidation changes. Besides, DECAF score is inconvenient for repeating assess because of delayed imaging changes in patients. Thus,prognostic indicators of in-hospital mortality in AECODP patients are not wellestablished till now. We need objective and easy predictors to predict the prognosis of AECOPD patients and maybe we could try interdisciplinary indexes.

Frailty is a clinical condition of decreased reserve and resistance to stressors. It prevails in older adults and cause increased vulnerability to many adverse health outcomes including death $[13,14]$. It is now recommended that older patients especially people are older than 70 years should have frailty assessments[15]. COPD patients are more likely to coexist with frailty. They share similar risk factors such as older ages and smoking. Common pathogenesis including inflammatory damage and endocrine dysregulation might influence the two diseases[16]. Hence, can we find an objective and easily measurable indicator of frailty to predict the mortality in AECOPD patients?

The frailty index based on routine laboratory tests (FI-Lab) is just such a comprehensive, objective and easily detectable index to quantify frailty. FI-Lab bases on the accumulation of health deficits[17] including 21 common blood tests(complete blood count, renal function, thyroid function, liver function, electrolytes, et al.)plus systolic and diastolic blood pressure. An Fl-Lab score is constructed by counting the number of deficits in a patient and dividing by the total number of deficits measured to produce a score between 0 and 1. A higher score indicates greater frailty. Rockwood[18] and Howlett [19] studies have confirmed that FI-Lab can quantify health and indicate adverse outcomes, including death, but most of the current prognostic studies of FI-Lab have focused on long-term care nursing home $[13,19,20]$, so what is the correlation between FI-Lab and the prognosis of older AECOPD inpatients? Therefore, we aim to investigate the prognostic value of FI-Lab in older Chinese AECOPD hospitalized patients.

\section{Methods}




\section{Study design and study participants}

A retrospective observational study was conducted at Zhenjiang First People's Hospital, Jiangsu Province, China, between September, 2016 and June, 2019. This is a tertiary teaching hospital of china. The study protocol was approved by the hospital ethics committee. All data were collected retrospectively from the hospital database. Due to the retrospective feature of the study design, informed consent was not obtained.

The study included patients aged 60 years or older who were admitted to respiratory or geriatrics department with a primary clinical diagnosis of AECOPD. A total of 1932 patients (1824 patients survived and 108 Patients died in the hospital) were screened in the study. We excluded patients hospitalized for secondary causes such as lung cancer, bronchiectasis, asthma, interstitial pulmonary disease, and active pulmonary tuberculosis. Each patient was enrolled in the study only once at their initial hospitalization. Patients with incomplete data-electrocardiograph, blood gas analysis $\llbracket$ chest X-ray or CT scan, and hematological data-were excluded. We also excluded patients with automatic discharge or transfer to another hospital.

After the above exclusion, there were 426 survivors and 98 non-survivors. A priori differences in patient characteristics between survivors and non-survivors may lead to biased estimates. In order to decrease this bias, we used propensity score matching (PSM) matching techniques[21]. We used a logistic regression model to estimate the propensity of participating in the two groups based on a set of observed covariates. PSM matching (1:1 matching) covariates included: age, gender, number of smokers, number of drinkers, history of comorbid diseases (including type 2 diabetes, hypertension, myocardial infarction or stroke). Finally, 77 survivors and 77 non-survivors were included in the study. The flow chart of subject inclusion is summarized in Figure 1.

\section{Definitions}

\section{COPD and AECOPD}

The COPD diagnosis was established by a consistent airflow obstruction on spirometry (FEV1/forced vital capacity $<0.70$ )[22]. The exacerbation of COPD (AECOPD) was defined as acute change in a patient's respiratory symptoms that is beyond normal variability, and that is sufficient to warrant a change in therapy[22].

\section{FI-Lab}

Frailty in this study is defined as "a medical syndrome with multiple causes and contributors that is characterized by diminished strength, endurance, and reduced physiologic function" [23].

Rockwood[18]and Howlett [19] developed an FI (the FI-Lab) of up to 23 variables based on 21 routine blood tests plus measured systolic and diastolic blood pressure based on deficit 
accumulation(Supplementary table 1). The Fl-Lab can meet several criterions about ideal biomarkers 刃influential to the pathogenesis, easy to measure, sensitive to changes, improved to intervention,

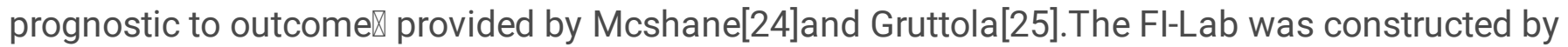
evaluating each variable as either 0 or 1 . ' 0 ' indicates that values are within the normal cut-offs but ' 1 ' indicates that values are outside of the normal cut-offs as deficits. An Fl-Lab score is constructed by counting the number of deficits and dividing by the total number of items tested to produce a score between 0 and 1. For example, a patient with deficits in six variables of the 23-item FI-Lab would have an FI-Lab score of 0.26 (6 divided by 23 ). A higher score indicates greater frailty.

An FI-Lab score was calculated only if more than $70 \%$ of the lab variables (items $16-23$ ) were available for a given. In this study, we use Fl-lab to evaluate frailty. We treated the FI-Lab score as a continuous variable as reported by previous studies and categorized the participants based on FI-Lab value into four grades: $<0.2,0.2-0.4,0.4-0.6$, and $>0.6$. We also tried to find out an optimal cutoff of the FI-Lab value to predict mortality.

\section{DECAF Score}

DECAF consists of five parameters: dyspnea (D), eosinopenia (E), consolidation (C), acidemia (A), and atrial fibrillation (F). Evaluating DECAF in AECOPD patients can help to predict mortality. DECAF is a commonly used predictor of AECOPD with a score range between 1 and 6 . A higher score indicates

poorer condition. A recent study reported that patients with a DECAF score of four or higher have a significant risk of mortality[6].Based on DECAF score『we categorized the participants into four grades: $\leq 2,3,4$, and $\geq 5$.

\section{Measurements}

Electronic data were collected from inpatient hospital database. Patients characteristics such as gender, age, smoking, alcohol consumption, comorbid diseases (diabetes, hypertension, myocardial infarction or stroke),length of stay, rehospitalization \and mortality were recorded. We extracted first blood test results and systolic pressure, diastolic blood pressure on admission.

Blood test results includes: complete blood count (total leukocyte, neutrophil, eosinophil, lymphocyte, platelet counts, mean platelet volume, red cell distribution width, hemoglobin); blood biochemical (total protein, albumin, aspartate aminotransferase, calcium, creatinine, urea, fasting blood glucose, alkaline phosphatase, phosphorus, potassium, sodium); thyroid function (thyroid stimulating hormone, thyroxine, free thyroxine); syphilis; hematopoietic raw materials (serum folate, vitamin B12); inflammatory markers including C-reactive protein(CRP), neutrophil-to-lymphocyte ratio(NLR), platelet-to- lymphocyte ratio(PLR);blood gas analysis.

\section{Statistical analysis}


Data were analyzed using IBM SPSS for Windows, Version 23.0 (IBM Corp, Armonk, NY). We compared survivors to non-survivors. The baseline difference between the groups was matched by PSM. The median with interquartile range was employed for nonparametric continuous variables, and mean \pm standard deviation was used for parametric continuous variables. Count and percentage were used when applicable. Mann-Whitney U-tests for nonparametric continuous variables or Student's t-tests for parametric continuous variables. Chi-square tests were employed for dichotomous variables. Logistic regression analysis was used to select the associated predictors of in-hospital mortality. Receiveroperating characteristic (ROC) curves were calculated to estimate the area under the ROC curve (AUCs) for FI-Lab and DECAF in relation to mortality. The comparison of the AUCs was performed using the DeLong method [26]. We applied the Youden index method to determine the optimal cutoffs of FI-Lab or DECAF for predicting mortality. A two-tailed P-value 0.05 was accepted as statistically significant.

\section{Results}

A total of 154 patients were included in the study. The mean age of these patients was $79.73 \pm 8.38$ years. There were $109(75.69 \%)$ men and $35(24.31 \%)$ women. The mean length of hospital days were $10.16 \pm 6.91$ days.

We compared the admission characteristics of survivors and non-survivors. Men were more common in both groups. Non-survivors had higher partial pressure of carbon dioxide $\left(\mathrm{PaCO}_{2}\right)$ and inflammatory markers (NLR, PLR, CRP). The characteristics and laboratory findings of survivors and non-survivors on admission are shown in Table 1.

We calculated FI-Lab for all patients. FI-Lab values were categorized into four grades: $<0.2,0.2-0.4,0.4-$

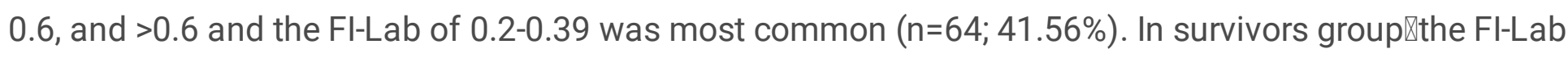

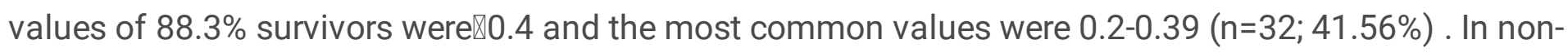
survivors group $\bigotimes$ the $\mathrm{FI}-\mathrm{Lab}$ values of $75.3 \%$ non-survivors were $\mathbb{0 . 4}$ and the most common values were 0.4-0.6 $(n=39 ; 50.65 \%)$. Mean FI-Lab of non-survivors was statistically higher than that of survivors $\bigotimes 0.51 \pm 0.13$ versus $0.29 \pm 0.10 \otimes P=0.000 \otimes($ Table 2$)$.

We also calculated DECAF scores for all patients. The DECAF scores were categorized into four grades: $\leq 2,3,4$, and $\geq 5$. A DECAF score of 4 was most common ( $n=53 ; 34.41 \%)$. The most common DECAF scores in non-survivors were $\geq 4(n=71 \otimes 92.21 \%)$ while the scores were $\mathbb{4}$ in survivors $(n=56 ; 72.73 \%)$. Mean DECAF Score of non-survivors was statistically higher than that of survivors $₫ 4.45 \pm 0.80$ versus $3.03 \pm 0.90 \rrbracket$ $P=0.000 \otimes$ (Table 2).

\section{Table 1 Characteristics and some laboratory findings of survivors and non-survivors on admission}




\begin{tabular}{|c|c|c|c|}
\hline Indexes & Survivors $\square n=77 \square$ & Non-survivors $\square \mathrm{n}=77 \square$ & P-value \\
\hline Age $\rrbracket$ years $\rrbracket$ & $79.38 \pm 8.14$ & $80.09 \pm 8.65$ & 0.207 \\
\hline Men $\otimes \square$ & 57ه74.0区 & $52 \bowtie 67.5 \rrbracket$ & 0.376 \\
\hline Current smokers $₫ \% \rrbracket$ & $45(58.4)$ & $40(51.9)$ & 0.418 \\
\hline Length of hospital days & $10.06 \pm 4.53$ & $10.26 \pm 8.70$ & 0.862 \\
\hline Current alcohol drinkers $₫ \% \rrbracket$ & $11(14.3)$ & 10(13) & 0.814 \\
\hline Systolic blood pressure $\ \mathrm{mmHg} \rrbracket$ & $136.65 \pm 22.63$ & $77.92 \pm 11.52$ & 0.136 \\
\hline Diastolic blood pressure $\ \mathrm{mmHg} \rrbracket$ & $77.92 \pm 11.52$ & $75.61 \pm 15.00$ & 0.285 \\
\hline $\mathrm{PaCO} 2(\mathrm{mmHg})$ & $51.13 \pm 13.48$ & $64.49 \pm 21.63$ & 0.000 \\
\hline \multicolumn{4}{|l|}{ Comorbidities $\square \%$} \\
\hline Diabetes & 12(15.6) & 16(20.8) & 0.664 \\
\hline Hypertension & $41(53.2)$ & $46(59.7)$ & 0.416 \\
\hline Stroke or Ischemic heart disease & 17(22.1) & 26(33.8) & 0.106 \\
\hline \multicolumn{4}{|l|}{ Complete blood count results } \\
\hline Leukocyte count $\triangle \times 10^{9} / \mathrm{L} \otimes$ & $7.66 \pm 3.87$ & $10.70 \pm 7.40$ & 0.002 \\
\hline Neutrophil count $\varangle \times 10^{9} / \mathrm{L} \rrbracket$ & $6.14 \pm 3.59$ & $9.36 \pm 7.08$ & 0.001 \\
\hline Lymphocyte count $₫ \times 10^{9} / \mathrm{L} \rrbracket$ & $1.14 \pm 0.78$ & $0.84 \pm 0.72$ & 0.016 \\
\hline Eosinophil count $\mathbb{X} \times 10^{9} / \mathrm{L} \rrbracket$ & $0.02(0.00,0.10)$ & $0(0,0.01)$ & 0.003 \\
\hline Platelet count $₫ \times 10^{9} / \mathrm{L} \rrbracket$ & $197.58 \pm 73.24$ & $185.27 \pm 86.34$ & 0.341 \\
\hline MPV (fl) & $10.31 \pm 1.32$ & $10.29 \pm 1.26$ & 0.906 \\
\hline RDW (\%) & $13.74 \pm 1.06$ & $15.36 \pm 2.89$ & 0.000 \\
\hline 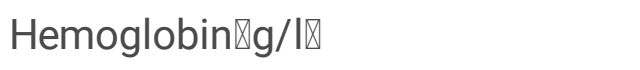 & $125.19 \pm 16.90$ & $107.90 \pm 23.46$ & 0.000 \\
\hline \multicolumn{4}{|l|}{ Inflammatory markers } \\
\hline NLR & $5.36(3.27,10.29)$ & $11.60(6.20,19.35)$ & 0.000 \\
\hline PLR & 191.25(132.20,288.27) & $245.0(147.9,406.65)$ & 0.047 \\
\hline CRP (mg/l) & $13.17(4.08,59.35)$ & $25.24(9.90,79.45)$ & 0.011 \\
\hline
\end{tabular}

Notes: neutrophil and lymphocyte ratio (NLR); red blood cell distribution (RDW); mean platelet volume (MPV); C-reactive protein (CRP), neutrophil-to-lymphocyte ratio (NLR); platelet-to-lymphocyte ratio (PLR); 
partial pressure of carbon dioxide $\left(\mathrm{PaCO}_{2}\right)$

Table 2 Fl-Lab and DECAF of survivors and non-survivors at the time of admission

\begin{tabular}{|c|c|c|c|}
\hline Values & Survivors $\square n=77 \square$ & Non-survivors $\square \mathrm{n}=77 \square$ & $P$-value \\
\hline \multicolumn{4}{|c|}{ 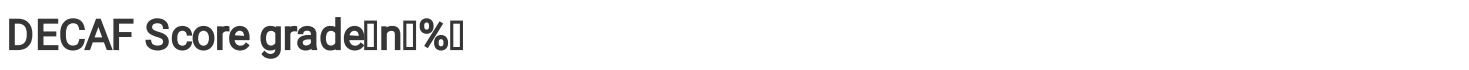 } \\
\hline$\leq 2$ & 24 & 1 & 0.000 \\
\hline 3 & 32 & 5 & 0.000 \\
\hline 4 & 17 & 36 & 0.000 \\
\hline$\geq 5$ & 4 & 35 & 0.000 \\
\hline Mean DECAF score & $3.03 \pm 0.90$ & $4.45 \pm 0.80$ & 0.000 \\
\hline \multicolumn{4}{|l|}{ Fl-Lab grade[n][\%] } \\
\hline$\varangle 0.2$ & $20 \rrbracket 25.97 \rrbracket$ & 0 & 0.000 \\
\hline $0.20-0.39$ & $48 \rrbracket 62.34 \rrbracket$ & 18 & 0.000 \\
\hline $0.40-0.6$ & $9 \rrbracket 11.68 \rrbracket$ & 39 & 0.000 \\
\hline$\geq 0.60$ & 1 & 19 & 0.000 \\
\hline Mean FI-Lab & $0.29 \pm 0.10$ & $0.51 \pm 0.13$ & 0.000 \\
\hline
\end{tabular}

Using the data shown in Table 1 and Table 2 , we included the variables -inflammatory markers $\triangle \mathrm{DECAF}$

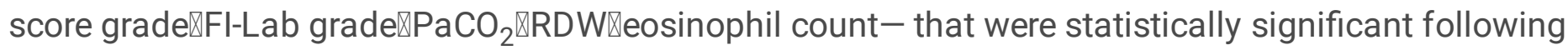
univariate analyses of survival and non-survival patients. The rest of the complete blood count parameters (leukocytes, neutrophils, hemoglobin, et al.) and blood biochemistry parameters are all part of the FI-Lab 21-item blood parameters and are not included in the logistic regression equation. Variables \age \gender, blood pressure, comorbidities, platelet count, et al.囚not statistically significant are also not included in the logistic regression equation.

Finally $\llbracket$ we included eight parameters including CRP, NLR, PLR, DECAF score grade $\mathbb{F I} \mathrm{L}$ ab grade, $\mathrm{PaCO}$, RDW, eosinophil count in the logistic regression model-method: Forward Stepwise (Likelihood Ratio). The results showed that high DECAF score grade and high FI-Lab grade were correlated with in-hospital mortality in AECOPD patients $(P=0.000)$, as shown in Table 3 .

ROC curves were calculated to estimate the AUCs for FI-Lab and DECAF in relation to mortality (Fig 2). The comparison of the AUCs was performed using the DeLong method. The AUCs were 0.906 for FI-Lab, 0.870 for DECAF with no statistically significant $\triangle P=0.2991 \rrbracket$. We applied the Youden index method to determine the optimal cutoffs of FI-Lab or DECAF in predicting mortality. When the FI-Lab was 0.4388 , the sensitivity, specificity and Youden index were $70.1 \%, 96.1 \%$ and 0.675 respectively. When DECAF score 
was 3.5 , the sensitivity, specificity and Youden index were $92.2 \%, 72.7 \%$, and 0.649 . FI-Lab has a slightly stronger screening ability than DECAF.

Table 3 Results of multivariate Logistic analysis

\begin{tabular}{|lllllll|}
\hline Variables & $\boldsymbol{\beta}$ & Sb & Wald $\mathbf{2}$ & Odds ratio & 95\%Cl囚lower-upper limit & $\boldsymbol{P}$-value \\
\hline Fl-Lab grade & 2.164 & 0.444 & 23.753 & 8.705 & $3.646-20.782$ & 0.000 \\
\hline DECAF score grade & 1.726 & 0.354 & 24.748 & 5.620 & $2.811-11.236$ & 0.000 \\
\hline
\end{tabular}

\section{Discussion}

This study is the first to compare the prognostic value of the FI-Lab and DECAF of older AECOPD inpatients in China. In contrast to the traditional use of respiratory indices to predict mortality in AECOPD, we used FI-Lab-an interdisciplinary index-to predict the risk of AECOPD patients. Our results suggested that both FI-Lab and DECAF can be successfully applied to predict in-hospital mortality. The FI-Lab appears to be slightly better than DECAF but with no statistical significance.

Our study also demonstrated that Mean FI-Lab and mean DECAF score in non-survivors were much higher than those of survivors. The inpatient mortality increased with increase in the FI-Lab value and DECAF score. The risk of in-hospital mortality may be indicated by the FI-Lab value $\geq 0.4388$ or the DECAF score $\geq 3.5$, respectively.

This study found that single indexes including RDW, CRP, and PaCO2 in non-survivors were significantly higher than those of survivors. Besides,eosinophil count in non-survivors were significantly lower than those of survivors. However, after logistic regression analysis, only FI-Lab grade and DECAF score grade were risk factors for death. This suggests that single indexes can associate with disease but show low prognostic value. Gu. et al [27] pointed out that no single biomarkers can be applied to the quantitative diagnosis and prognosis of AECOPD, so we should seek new comprehensive biomarkers that not only limited to inflammatory markers or respiratory markers. Both of FI-Lab grade and DECAF Score are comprehensive biomarkers, we compared their differences.

Nafae et al. [28] reported that DECAF was a significantly predictor of in-hospital mortality and its AUCs was 0.870 . The AUCs of DECAF of our study was also 0.870 occasionally. Many studies[5, 12] have shown its strong value in predicting mortality in AECOPD patients to be superior to other respiratory scores including the APACHE score, CAPS score, CURB $₫ 65$ score, and BAP $₫ 65$ score. However,the DECAF indicator still has some relative weaknesses. Firstly, our study suggests that DECAF has high sensitivity but low specificity relatively (When DECAF cutoff score was 3.5 , the sensitivity and specificity were $92.2 \%$, $72.7 \%$, and Youden index 0.649).A study also shows the sensitivity of the DECAF score for predicting mortality in AECOPD patients was even $100 \%$ but specificity was poor囚34.1\%囚[29]. Secondly, the DECAF consists of five comprehensive and complicated parameters and covers clinical, serological, radiological and electrocardiographic scale. HencedDECAF score is inconvenient for repeated testing because imaging 
changes are relatively slow in patients. Besides $\square$ the DECAF score is somewhat subjective, especially in evaluating the degree of dyspnea. Finally, pulmonary consolidation of image tests often suggest pneumonia, and there is a debate to judge whether AECOPD includes AECOPD with pneumonia.

The association between frailty and mortality has been well established[15]. Based on the fact that COPD and frailty share a common pathogenesis and that co-morbidity is more common in older patients[30, 31]. The Frailty Index Based on Laboratory Tests (FI-Lab) used in this paper is a 23-item indicator (21 hematological indices plus systolic and diastolic blood pressure) program proposed by Howlett SE et al[18, 19, 32]. The FI-Lab has been widely used to objectively assess the degree of frailty. We found that the FI-Lab values were significantly higher in the non-survivors than those in the survivors.FI-Lab was significantly correlated with the mortality with an AUC area of 0.906 , suggesting that the predictive value of FI-Lab is slightly more reliable than DECAF(AUCs of 0.870). FI-Lab is objective to assess, easy to measure, and convenient to repeat. When the FI-Lab was 0.4388 , the specificity for predicting mortality was $96.1 \%$ and was much higher than DECAF score. However,although the specificity of FI-Lab reached $96.1 \%$, its sensitivity was still poor】only $70.1 \% \bigotimes$. Therefore, we still need to explore a better predictor for future research. Maybe we can get a more desirable indicator with good sensitivity and specificity through a combination of clinical, imaging and laboratory tests.

This study has some limitations. First,this is a retrospective study, we need a prospective cohort study in the future to conform the conclusion. Second, although we screened a large number of cases (total cases are1932,the final sample size of each group is relatively small(77 for each group). Third, because this was a retrospective study, it was not able to analyze some critical outcomes in detail including causes of death, falls during hospitalization, quality of life, incidence of acute cardiovascular events, and social support. Fourth, it may be time-consuming to determine the abnormalities of the 23 items. If the FI-Lab can be coded into a program (or an App) and integrated into hospital information systems (HIS) and then it can be calculated automatically. Finally, the sensitivity of FI-Lab is still low, we should treat prognostic value of Fl-Lab prudently.

In conclusion, the FI-Lab is a simple, effective and objective indicator and can be quick to help clinicians to assess in-hospital mortality of AECOPD patients. Dynamic monitoring of the FI-Lab may be of great clinical value in understanding patient progression and predicting the risk of death in AECOPD patients.

\section{Declarations}

\section{Authors' contributions}

Jinjin Gu and Qiang Liu designed and performed this study. Jinjin Gu, Lijie Zheng and Qiang Liu gave conceptual advice and contributed significantly to the data analyses, interpretion of the results, and edited the paper. Jinjin Gu wrote the paper. All authors read and approved the final manuscript.

\section{Funding}


The project was supported by a research grant by hospital-level scientific fund of Zhenjiang First People's Hospital (Y2019006).

\section{Availability of data and materials}

All data and materials can be accessed via Jinjin Gu and Qiang Liu.

\section{Ethics approval and consent to participate}

The study protocol was approved by the Hospital ethics committee. All data were collected retrospectively from the hospital database. Due to the retrospective nature of the study design, informed consent was not obtained.

\section{Consent for publication}

All authors gave consent for the publication.

\section{Competing interests}

All the authors declare no conflicts of interest in this work.

\section{Author details}

${ }^{1}$ Department of Geriatrics, Zhenjiang First People's Hospital, Zhenjiang 212002, Jiangsu Province, China;

2 Department of Laboratory Medicine, Zhenjiang First People's Hospital, Zhenjiang 212002, Jiangsu Province, China $\otimes^{3}$ Department of Laboratory Medicine, Affiliated Hospital of Jiangsu University, Zhenjiang 212001, Jiangsu Province, China.

\section{References}

1. Woodruff PG, Agusti A, Roche N, Singh D, Martinez FJ. Current concepts in targeting chronic obstructive pulmonary disease pharmacotherapy: making progress towards personalised management. Lancet. 2015;385:1789-98.

2. Tamer, Abdullah, Helmy, Ayman, Ibrahim, Baess, et al. Role of C-reactive protein and interleukin-6 in predicting the prognosis of ICU-admitted patients with acute exacerbation of COPD. Egyptian Journal of Chest Diseases \& Tuberculosis. 2014.

3. Duman D, Aksoy E, Agca MC, Kocak ND, Ozmen I, Akturk UA, et al. The utility of inflammatory markers to predict readmissions and mortality in COPD cases with or without eosinophilia. Int $J$ Chron Obstruct Pulmon Dis. 2015;10:2469-78.

4. Leuzzi G, Galeone C, Taverna F, Suatoni P, Morelli D, Pastorino U. C-reactive protein level predicts mortality in COPD: a systematic review and meta-analysis. Eur Respir Rev. 2017;26.

5. Steer J, Gibson J, Bourke SC. The DECAF Score: predicting hospital mortality in exacerbations of chronic obstructive pulmonary disease. Thorax. 2012;67:970-6. 
6. Memon MA, Faryal S, Brohi N, Kumar B. Role of the DECAF Score in Predicting In-hospital Mortality in Acute Exacerbation of Chronic Obstructive Pulmonary Disease. Cureus. 2019;11:e4826.

7. K P, Surapaneni S, N S. DECAF score for assessing prognosis in Acute Exacerbation of COPD. J Assoc Physicians India. 2020;68:82.

8. Rahimirad S, Ghafari M, Ansarin K, Rashidi F, Rahimi-Rad MH. Elevated red blood cell distribution width predicts mortality in acute exacerbation of COPD. Pneumologia. 2016;65:85-9.

9. Epstein D, Nasser R, Mashiach T, Azzam ZS, Berger G. Increased red cell distribution width: A novel predictor of adverse outcome in patients hospitalized due to acute exacerbation of chronic obstructive pulmonary disease. Respir Med. 2018;136:1-7.

10. Wu CW, Lan CC, Hsieh PC, Tzeng IS, Wu YK. Role of peripheral eosinophilia in acute exacerbation of chronic obstructive pulmonary disease. World J Clin Cases. 2020;8:2727-37.

11. Yao C, Liu X, Tang Z. Prognostic role of neutrophil-lymphocyte ratio and platelet-lymphocyte ratio for hospital mortality in patients with AECOPD. Int J Chron Obstruct Pulmon Dis. 2017;12:2285-90.

12. Huang $\mathrm{Q}, \mathrm{He} \mathrm{C}$, Xiong $\mathrm{H}$, Shuai $\mathrm{T}$, Zhang $\mathrm{C}$, Zhang $\mathrm{M}$, et al. DECAF score as a mortality predictor for acute exacerbation of chronic obstructive pulmonary disease: a systematic review and metaanalysis. BMJ Open. 2020;10:e037923.

13. Rockwood K, Song X, Macknight C, Bergman H, Hogan DB, Mcdowell I, et al. A global clinical measure of fitness and frailty in elderly people. Cmaj Canadian Medical Association Journal. 2005;173:489-95.

14. Kizilarslanoglu MC, Civelek R, Kilic MK, Sumer F, Varan HD, Kara O, et al. Is frailty a prognostic factor for critically ill elderly patients. Aging Clinical \& Experimental Research. 2017;29:1-9.

15. Morley JE, Vellas B, van Kan GA, Anker SD, Bauer JM, Bernabei R, et al. Frailty consensus: a call to action. J Am Med Dir Assoc. 2013;14:392-7.

16. Fragoso CAV, Enright PL, McAvay G, Van Ness PH, Gill TM. Frailty and Respiratory Impairment in Older Persons. Am J Med. 2012;125:0-86.

17. Searle SD, Mitnitski A, Gahbauer EA, Gill TM, Rockwood K. A standard procedure for creating a frailty index. BMC Geriatr. 2008;8:24.

18. Rockwood K, McMillan M, Mitnitski A, Howlett SE. A Frailty Index Based on Common Laboratory Tests in Comparison With a Clinical Frailty Index for Older Adults in Long-Term Care Facilities. J Am Med Dir Assoc. 2015;16:842-7.

19. Howlett SE, Rockwood MR, Mitnitski A, Rockwood K. Standard laboratory tests to identify older adults at increased risk of death. BMC Med. 2014;12:171.

20. Yang M, Zhuo Y, Hu X, Xie L. Predictive validity of two frailty tools for mortality in Chinese nursing home residents: frailty index based on common laboratory tests (FI-Lab) versus FRAIL-NH. Aging Clin Exp Res. 2018;30:1445-52.

21. Rosenbaum PR, Rubin DB. The Central Role of the Propensity Score in Observational Studies for Causal Effects. Biometrika. 1983;70:41-55-. 
22. Vogelmeier CF, Criner GJ, Martinez FJ, Anzueto A, Barnes PJ, Bourbeau J, et al. Global Strategy for the Diagnosis, Management and Prevention of Chronic Obstructive Lung Disease 2017 Report: GOLD Executive Summary. Respirology. 2017;22:575-601.

23. Clegg A, Young J, lliffe S, Rikkert MO, Rockwood K. Frailty in elderly people. Lancet. 2013;381:752-62.

24. Mcshane LM, Altman DG, Willi S. Identification of clinically useful cancer prognostic factors: what are we missing. J Natl Cancer Inst. 2005;97:1023-5.

25. Gruttola VGD, Clax P, Demets DL, Downing GJ, Ellenberg SS, Friedman L, et al. Considerations in the Evaluation of Surrogate Endpoints in Clinical Trials: Summary of a National Institutes of Health Workshop. J Natl Cancer Inst. 2001;22:485-502.

26. DeLong ER, DeLong DM, Clarke-Pearson DL. Comparing the areas under two or more correlated receiver operating characteristic curves: a nonparametric approach. Biometrics. 1988;44:837-45.

27. Gu Y. [Biomarker in acute exacerbation of chronic obstructive pulmonary disease]. Zhonghua Jie He He Hu Xi Za Zhi. 2014;37:247-9.

28. Nafae R, Embarak S, Gad DM. Value of the DECAF score in predicting hospital mortality in patients with acute exacerbation of chronic obstructive pulmonary disease admitted to Zagazig University Hospitals, Egypt. Egyptian Journal of Chest Diseases and Tuberculosis. 2015;64:35-40-.

29. Sangwan V, Chaudhry D, Malik R. Dyspnea, Eosinopenia, Consolidation, Acidemia and Atrial Fibrillation Score and BAP-65 Score, Tools for Prediction of Mortality in Acute Exacerbations of Chronic Obstructive Pulmonary Disease: A Comparative Pilot Study. Indian J Crit Care Med. 2017;21:671-7-.

30. Gale NS, Albarrati AM, Munnery MM, Hubbard RE, Tal-Singer R, Cockcroft JR, et al. Frailty: A global measure of the multisystem impact of COPD. Chron Respir Dis. 2018;15:347-55.

31. Bernabeu-Mora R, Oliveira-Sousa SL, Sánchez-Martínez MP, García-Vidal JA, Gacto-Sánchez M, Medina-Mirapeix F. Frailty transitions and associated clinical outcomes in patients with stable COPD: A longitudinal study. PLoS One. 2020;15:e0230116.

32. Blodgett JM, Theou O, Howlett SE, Wu FCW, Rockwood K. A frailty index based on laboratory deficits in community-dwelling men predicted their risk of adverse health outcomes. Age \& Ageing. 2016;45:afw054.

\section{Supplementary Information}

\section{Supplementary Table 1 Clinical and laboratory data used to construct the FI-Lab[18, 19]}




\begin{tabular}{|c|c|c|}
\hline Variable & Low cut-off & High cut-off \\
\hline Albumin (g/L) & 32 & 45 \\
\hline AST (SGOT; IU/L) & 8 & 33 \\
\hline supine systolic $(\mathrm{mmHg})$ & 90 & 140 \\
\hline supine diastolic (mmHg) & 60 & 90 \\
\hline Calcium (mM) & 2.3 & 2.7 \\
\hline Creatinine $(\mu \mathrm{M})$ & 53 & 106 \\
\hline Folate (nM) & 11 & 57 \\
\hline Folate, RBC (nM) & 376 & 1450 \\
\hline Glucose, fasting (mM) & 3.9 & 6.1 \\
\hline Hemoglobin $(g / L)^{a}$ & $135 / 120$ & $180 / 160$ \\
\hline MPV (fL) & 80 & 96 \\
\hline alkaline phosphatase (IU/L) & 20 & 130 \\
\hline inorganic phosphorus (mM) & 0.74 & 1.52 \\
\hline Potassium (mM) & 3.8 & 5 \\
\hline Protein, total (g/L) & 60 & 78 \\
\hline Sodium (mM) & 136 & 142 \\
\hline $\mathrm{TSH}(\mu \mathrm{IU} / \mathrm{L})$ & 0.5 & 5 \\
\hline Thyroxine (T4; nM) & 71 & 161 \\
\hline T4, Free $(\mathrm{pM})$ & 12 & 30 \\
\hline Urea (mM) & 2.9 & 8.2 \\
\hline syphilis & 0 & 0 \\
\hline Vitamin B12 & 118 & 701 \\
\hline 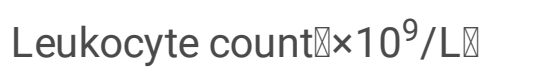 & $1.8 \times 10^{9}$ & $7.8 \times 10^{9}$ \\
\hline
\end{tabular}

a Note that normal references values for hemoglobin differed between the sexes so for women, the low cut-off was $120 \mathrm{~g} / \mathrm{L}$ and the high cut-off was $160 \mathrm{~g} / \mathrm{L}$.

Notes: aspartate aminotransferase(AST); thyroid-stimulating hormone(TSH); mean platelet volume (MPV). 


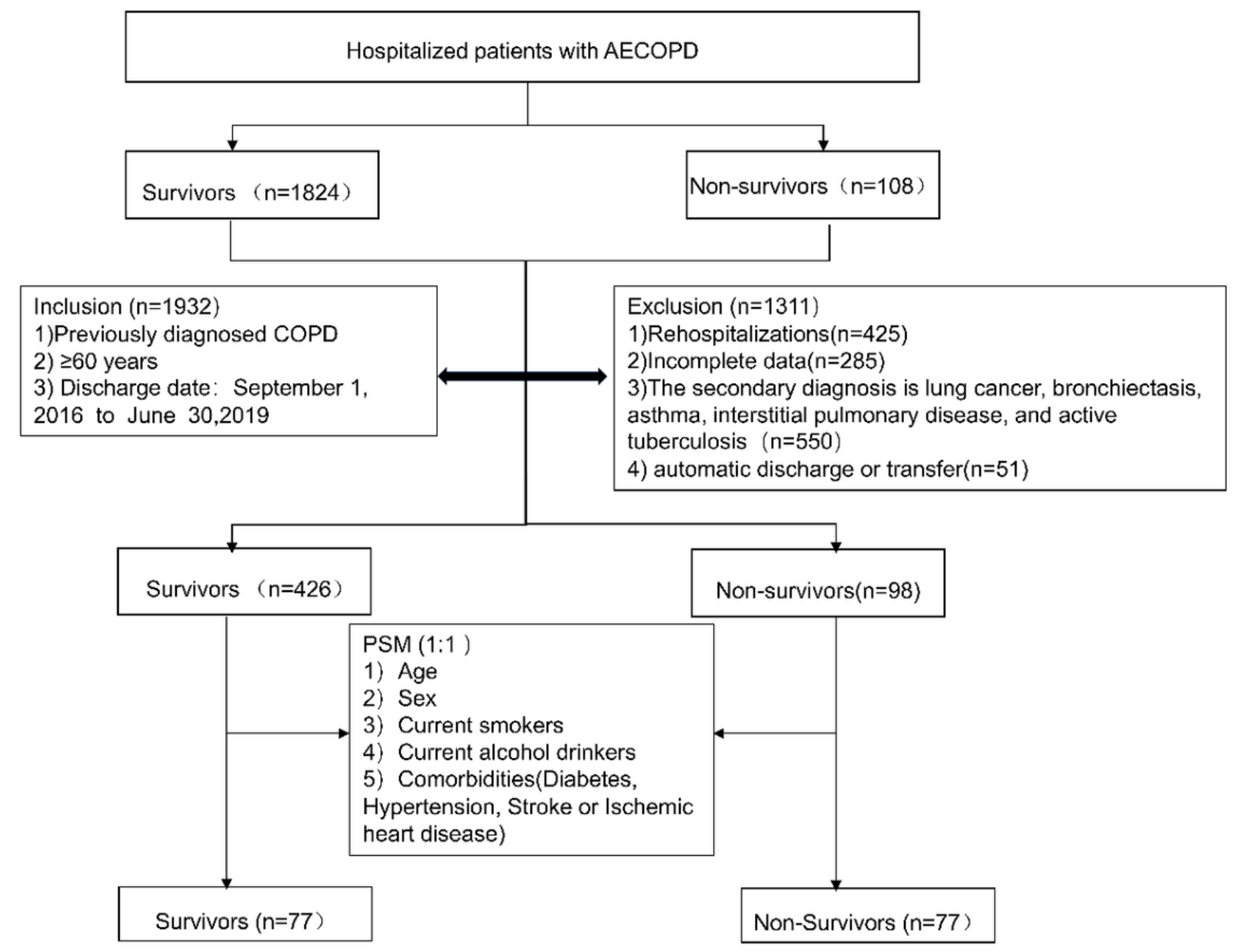

\section{Figure 1}

Flow chart of patient admission 


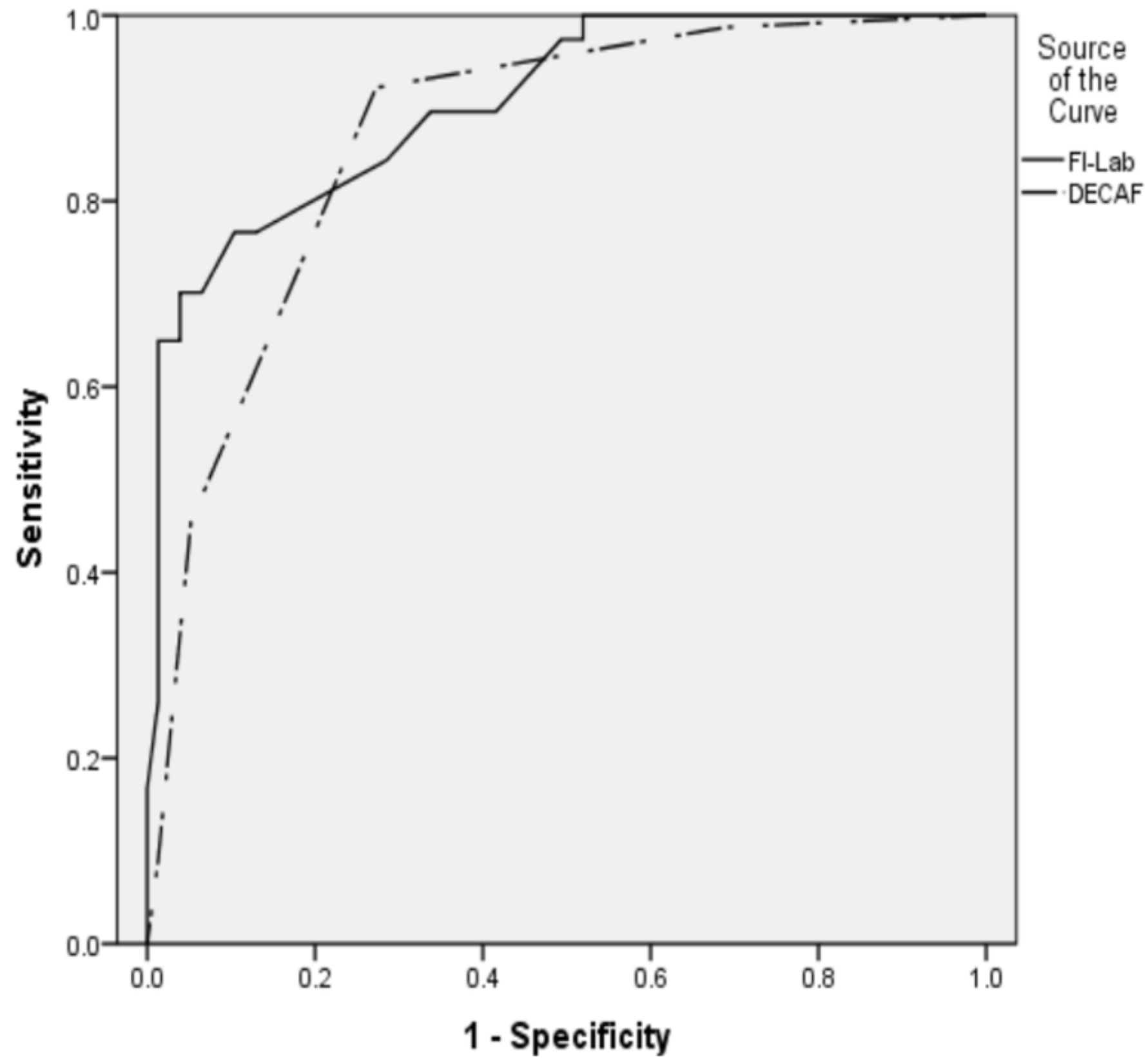

Figure 2

ROC curves for FI-Lab and DECAF 